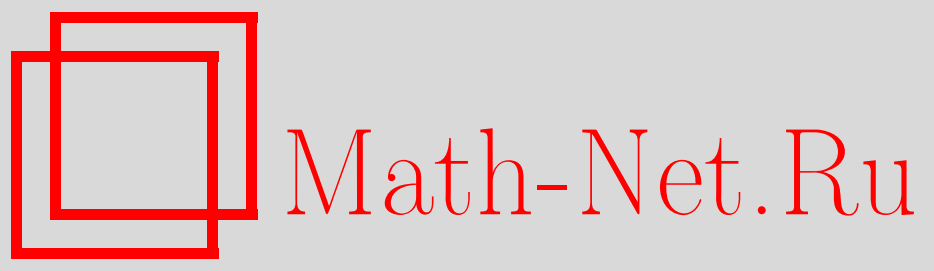

Б. Г. Конопельченко, Л. Мартинес Алонсо, Е. Медина, Сингулярные секторы однослойной системы Бенни; бездисперсионные системы Тоды и соотношения между ними, ТМФ, 2011, том 168, номер 1, 125-137

DOI: https://doi.org/10.4213/tmf6668

Использование Общероссийского математического портала Math-Net.Ru подразумевает, что вы прочитали и согласны с пользовательским соглашением http://www.mathnet.ru/rus/agreement

Параметры загрузки:

IP : 54.172 .240 .79

26 апреля 2023 г., 14:12:04

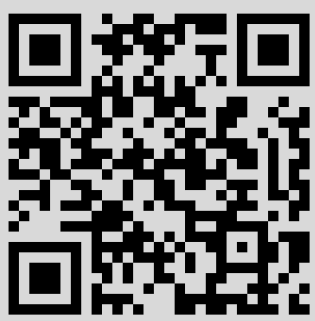




\title{
СИНГУЛЯРНЫЕ СЕКТОРЫ ОДНОСЛОЙНОЙ СИСТЕМЫ БЕННИ; БЕЗДИСПЕРСИОННЫЕ СИСТЕМЫ ТОДЫ И СООТНОШЕНИЯ МЕЖДУ НИМИ
}

\begin{abstract}
Представлено полное описание сингулярных секторов однослойной системы Бенни (классическое уравнение длинных волн) и бездисперсионной системы Тоды. Основным инструментом анализа являются ассоциированные уравнения Эйлера-Пуассона-Дарбу $E(1 / 2,1 / 2)$ и $E(-1 / 2,-1 / 2)$. Представлен полный список решений для однослойной системы Бенни, зависящей от двух параметров и принадлежащей сингулярному сектору. Обсуждается соотношение между уравнениями Эйлера-Пуассона-Дарбу $E(\varepsilon, \varepsilon)$ с противоположным знаком $\varepsilon$.
\end{abstract}

Ключевые слова: критические точки, сингулярный сектор, уравнение ЭйлераПуассона-Дарбу.

\section{1. ВВЕДЕНИЕ}

Однослойная система Бенни (классическое уравнение длинных волн)

$$
u_{t}+u u_{x}+v_{x}=0, \quad v_{t}+(u v)_{x}=0
$$

и бездисперсионное уравнение Тоды $v_{x x}=(\ln v)_{t t}$ или, эквивалентно, система

$$
u_{t}+v_{x}=0, \quad v_{t}+v u_{x}=0
$$

представляют собой две важные интегрируемые системы гидродинамического типа (см., например, работы [1], [2]). Однослойная система Бенни описывает длинные волны в мелкой воде со свободной поверхностью в гравитационном поле. Она является бездисперсионным пределом нелинейного уравнения Шредингера (НУШ) [3]. Недавно однослойная система Бенни $B(1)$ стала важным элементом анализа универсальности критического поведения для нелинейных уравнений [4]. Бездисперсионное уравнение Тоды представляет собой $(1+1)$-мерный вариант уравнения

*Dipartimento di Fisica, Universitá del Salento and Sezione INFN, Lecce, Italy. E-mail: Boris.Konopeltchenko@le.infn.it

${ }^{\dagger}$ Departamento de Física Teórica II, Universidad Complutense, Madrid, Spain

${ }^{\ddagger}$ Departamento de Matemáticas, Universidad de Cádiz, Puerto Real, Cádiz, Spain 
Бойера-Финли из общей теории относительности [5]. Оно появляется в различных задачах механики жидкостей (см., например, работу [6]). Кроме того, известно [7], что уравнения годографа бездисперсионной иерархии Тоды определяют в переделе больших $N$ эрмитову модель в теории случайных матриц. В общем, эти две системы представляют собой превосходную лабораторию для изучения свойств интегрируемых систем гидродинамического типа.

В настоящей работе мы анализируем структуру системы уравнений годографа $B(1)$-иерархии и бездисперсионной иерархии Тоды в терминах их римановых инвариантов. Соответствующие решения годографа описывают критические точки

$$
\frac{\partial W}{\partial \beta_{i}}=0, \quad i=1,2,
$$

функции $W=W\left(\boldsymbol{t}, \beta_{1}, \beta_{2}\right)$, которая линейно зависит от координат $\boldsymbol{t}$, где $\boldsymbol{t}$ - параметры потока для иерархии Бенни и бездисперсионной иерархии Тоды соответственно, и удовлетворяют уравнению Эйлера-Пуассона-Дарбу (ЭПД) $E(\varepsilon, \varepsilon)[8]:$

$$
\left(\beta_{1}-\beta_{2}\right) \frac{\partial^{2} W}{\partial \beta_{1} \partial \beta_{2}}=\varepsilon\left(\frac{\partial W}{\partial \beta_{1}}-\frac{\partial W}{\partial \beta_{2}}\right),
$$

где для системы Бенни $\varepsilon=1 / 2$, а для бездисперсионной системы Тоды $\varepsilon=-1 / 2$. Уравнение (4) и его многомерный вариант давно известны в классической геометрии [8]. Его применимость к теории уравнений Уизема была недавно показана в работах [9]-[11]. В настоящей работе мы будем использовать классические обозначения $E(\varepsilon, \mu)$ для уравнений ЭПД, предложенные в работе [8], где также рассматривались эти уравнения с различными $\varepsilon$ и $\mu$.

Если обозначить через $\mathcal{M}$ множество решений $(\boldsymbol{t}, \boldsymbol{\beta}), \beta_{1} \neq \beta_{2}$, уравнений годографа (3), можно выделить регулярный и сингулярный секторы в $\mathcal{M}$ :

$$
\mathcal{M}=\mathcal{M}^{\text {reg }} \cup \mathcal{M}^{\text {sing }},
$$

такие, что для заданных $(\boldsymbol{t}, \boldsymbol{\beta}) \in \mathcal{M}(\boldsymbol{t}, \boldsymbol{\beta}) \in \mathcal{M}^{\mathrm{reg}}$, если $\operatorname{det}\left(\partial^{2} W(\boldsymbol{t}, \boldsymbol{\beta}) / \partial \beta_{i} \partial \beta_{j}\right) \neq 0$, и $(\boldsymbol{t}, \boldsymbol{\beta}) \in \mathcal{M}^{\text {sing }}$, если $\operatorname{det}\left(\partial^{2} W(\boldsymbol{t}, \boldsymbol{\beta}) / \partial \beta_{i} \partial \beta_{j}\right)=0$. Элементы из $\mathcal{M}^{\text {reg }}$ соответствуют случаю, когда система (3) однозначно разрешима, и тем самым определено единственное решение $\boldsymbol{\beta}(\boldsymbol{t})$. Сингулярный класс $\mathcal{M}^{\text {sing }}$ представляет вырожденные критические точки функции $W$ - точки, в которых неявные решения $\boldsymbol{\beta}(\boldsymbol{t})$ уравнений годографа демонстрируют поведение "градиентной катастрофы". Как будет показано в настоящей работе, уравнения ЭПД важны при анализе структуры $\mathcal{M}^{\text {sing }}$. В качестве иллюстации общего результата представлен полный список решений однослойной иерархии Бенни из $\mathcal{M}^{\text {sing }}$, зависящих от двух параметров. Кроме того, рассматриваются соотношения между уравнениями ЭПД с противоположными значениями $a$ и уравнениями ЭПД для симметрий и плотностей интегралов движения для интегрируемых систем гидродинамического типа.

\section{2. ОДНОСЛОЙНАЯ ИЕРАРХИЯ БЕННИ И ЕЕ СИНГУЛЯРНЫЙ СЕКТОР}

Система $B(1)(1)$ представляет собой член бездисперсионной интегрируемой иерархии деформаций кривой (см., например, работы [12], [13])

$$
p^{2}=\left(\lambda-\beta_{1}\right)\left(\lambda-\beta_{2}\right),
$$


где $u=-\left(\beta_{1}+\beta_{2}\right), v=\left(\beta_{1}+\beta_{2}\right)^{2} / 4$. Потоки $\boldsymbol{\beta}(\boldsymbol{t})$ характеризуются следующим условием: существует семейство функций $S(\lambda, \boldsymbol{t}, \boldsymbol{\beta})$, удовлетворяющих уравнениям

$$
\partial_{t_{n}} S(\lambda, \boldsymbol{t}, \boldsymbol{\beta}(\boldsymbol{t}))=\Omega_{n}(\lambda, \boldsymbol{\beta}(\boldsymbol{t})), \quad n \geqslant 1
$$

где

$$
\Omega_{n}(\lambda, \boldsymbol{\beta})=\left(\frac{\lambda^{n}}{\sqrt{\left(\lambda-\beta_{1}\right)\left(\lambda-\beta_{2}\right)}}\right)_{\oplus} \sqrt{\left(\lambda-\beta_{1}\right)\left(\lambda-\beta_{2}\right)} .
$$

Здесь $\oplus$ обозначает стандартную проекцию на положительные степени $\lambda$. Функции $S$, удовлетворяющие уравнениям (6), известны в теории бездисперсионных интегрируемых систем как функиии действия [14]. Заметим, что при $n=1$ эти уравнения можно записать в виде

$$
p=\frac{\partial S}{\partial x}, \quad x:=t_{1}
$$

так что система (6) эквивалентна системе

$$
\partial_{t_{n}} p=\partial_{x} \Omega_{n}
$$

В терминах инвариантов Римана $\boldsymbol{\beta}$ ее можно переписать в гидродинамическом виде:

$$
\partial_{t_{n}} \beta_{i}=\left.\left(\frac{\lambda^{n}}{\sqrt{\left(\lambda-\beta_{1}\right)\left(\lambda-\beta_{2}\right)}}\right)_{\oplus}\right|_{\lambda=\beta_{i}} \partial_{x} \beta_{i}, \quad i=1,2 .
$$

$t_{2}$-Поток этой иерархии является $B(1)$-системой $(1)\left(t:=t_{2}\right)$ :

$$
\left\{\begin{array}{l}
\partial_{t} \beta_{1}=\frac{1}{2}\left(3 \beta_{1}+\beta_{2}\right) \beta_{1 x} \\
\partial_{t} \beta_{2}=\frac{1}{2}\left(3 \beta_{2}+\beta_{1}\right) \beta_{2 x}
\end{array}\right.
$$

При $v>0$ B(1)-система является гиперболической, а при $v<0$ - эллиптической.

В работе [15] было доказано, что система (3) для критических точек функции

$$
W(\boldsymbol{t}, \boldsymbol{\beta}):=\oint_{\gamma} \frac{d \lambda}{2 i \pi} \frac{V(\lambda, \boldsymbol{t})}{\sqrt{\left(\lambda-\beta_{1}\right)\left(\lambda-\beta_{2}\right)}},
$$

где $V(\lambda, \boldsymbol{t})=\sum_{n \geqslant 1} t_{n} \lambda^{n}$, представляет собой систему уравнений годографа для иерархии Бенни. Более того, функция действия для соответствующих решений имеет вид

$$
S(\lambda, \boldsymbol{t}, \boldsymbol{\beta})=\sum_{n \geqslant 1} t_{n} \Omega_{n}(\lambda, \boldsymbol{\beta})=h(\lambda, \boldsymbol{t}, \boldsymbol{\beta}) \sqrt{\left(\lambda-\beta_{1}\right)\left(\lambda-\beta_{2}\right)}
$$

где

$$
h(\lambda, \boldsymbol{t}, \boldsymbol{\beta}):=\left(\frac{V(\lambda, \boldsymbol{t})}{\sqrt{\left(\lambda-\beta_{1}\right)\left(\lambda-\beta_{2}\right)}}\right)_{\oplus} .
$$

Очевидно, что функция $W$ удовлетворяет уравнению ЭПД $E(1 / 2,1 / 2)$. 
В явной записи $W$ представляет собой ряд

$$
\begin{gathered}
W=\frac{x}{2}\left(\beta_{1}+\beta_{2}\right)+\frac{t_{2}}{8}\left(3 \beta_{1}^{2}+2 \beta_{1} \beta_{2}+3 \beta_{2}^{2}\right)+\frac{t_{3}}{16}\left(5 \beta_{1}^{3}+3 \beta_{1}^{2} \beta_{2}+3 \beta_{1} \beta_{2}^{2}+5 \beta_{2}^{3}\right)+ \\
+\frac{t_{4}}{128}\left(35 \beta_{1}^{4}+20 \beta_{1}^{3} \beta_{2}+18 \beta_{1}^{2} \beta_{2}^{2}+20 \beta_{1} \beta_{2}^{3}+35 \beta_{2}^{4}\right)+\cdots
\end{gathered}
$$

Уравнения годографа (3) при $t_{n}=0$ для $n \geqslant 5$ принимают вид

$$
\begin{aligned}
8 x & +4 t_{2}\left(3 \beta_{1}+\beta_{2}\right)+3 t_{3}\left(5 \beta_{1}^{2}+2 \beta_{1} \beta_{2}+\beta_{2}^{2}\right)+ \\
& +\frac{t_{4}}{8}\left(140 \beta_{1}^{3}+60 \beta_{1}^{2} \beta_{2}+18 \beta_{1} \beta_{2}^{2}+20 \beta_{2}^{2}\right)=0, \\
8 x & +4 t_{2}\left(\beta_{1}+3 \beta_{2}\right)+3 t_{3}\left(\beta_{1}^{2}+2 \beta_{1} \beta_{2}+5 \beta_{2}^{2}\right)+ \\
& +\frac{t_{4}}{8}\left(140 \beta_{2}^{3}+60 \beta_{2}^{2} \beta_{1}+18 \beta_{2} \beta_{1}^{2}+20 \beta_{1}^{2}\right)=0 .
\end{aligned}
$$

Подробный анализ уравнений (14) будет представлен в разделе 3 , а сейчас мы сделаем два наблюдения. Первое состоит в том, что формулы (14) указывают на возможную альтернативную интерпретацию времен $t_{2}, t_{3}, t_{4}, \ldots B(1)$-иерархии. А именно, полагая $t_{2}=0$ в формулах (14), мы видим, что $t_{3}$ и $t_{4}$ являются параметрами, появляющимися в начальных данных $\beta_{1}\left(x, t_{2}=0\right)$ и $\beta_{2}\left(x, t_{2}=0\right)$. Таким образом, можно рассматривать уравнения годографа (3) (в частности, уравнения (14)) как уравнения, описывающие временну́ю эволюцию семейств начальных данных для $B(1)$-системы, параметризованной переменными $t_{3}, t_{4}, t_{5}, \ldots$

Второе наблюдение касается эллиптического варианта $B(1)$-системы. В этом случае $\beta_{2}=\bar{\beta}_{1}$, и система (10) сводится к одному уравнению

$$
\partial_{t} \beta=\frac{1}{2}(3 \beta+\bar{\beta}) \beta_{x}, \quad t:=t_{2}, \quad \beta:=\beta_{1} .
$$

Это уравнение эквивалентно нелинейному уравнению Бельтрами

$$
\beta_{\bar{z}}=\frac{2 i-3 \beta-\bar{\beta}}{2 i+3 \beta+\bar{\beta}} \beta_{z},
$$

где $z=x+i t$. Этот факт указывает на то, что теория квазиконформных отображений (см., например, работу [16]) может быть актуальной для анализа свойств эллиптической $B(1)$-системы $(1)(v<0)$. Поэтому ввиду того, что эллиптическая $B(1)$-система представляет собой квазиклассический предел [3] фокусирующего НУШ

$$
i \epsilon \psi_{t}+\frac{\epsilon^{2}}{2} \psi_{x x}+|\psi|^{2} \psi=0
$$

где $\psi=A e^{i S / \epsilon}, u=(\epsilon /(2 i))\left(\psi_{x} / \psi-\bar{\psi}_{x} / \bar{\psi}\right), v=-|\psi|^{2}$ и $\epsilon \rightarrow 0$, квазиконформное отображение также может быть полезным при исследовании предела малой дисперсии фокусирующего НУШ (ср. с работой [4]).

Для анализа сингулярного сектора однослойной иерархии Бенни заметим сначала, что согласно уравнению ЭПД при заданных $(\boldsymbol{t}, \boldsymbol{\beta}) \in \mathcal{M}$ как следствие формулы (4) имеем

$$
\frac{\partial^{2} W}{\partial \beta_{1} \partial \beta_{2}}=0 .
$$


Следовательно,

$$
\operatorname{det}\left(\frac{\partial^{2} W}{\partial \beta_{i} \partial \beta_{j}}\right)=\frac{\partial^{2} W}{\partial \beta_{1}^{2}} \frac{\partial^{2} W}{\partial \beta_{2}^{2}}
$$

Таким образом получаем

ПрЕДЛОЖЕНИЕ 1. При заданном $(\boldsymbol{t}, \boldsymbol{\beta}) \in \mathcal{M}$ :

1) $(\boldsymbol{t}, \boldsymbol{\beta}) \in \mathcal{M}^{\text {reg }}$, если и только если $\partial^{2} W / \partial \beta_{1}^{2} \neq 0$ и $\partial^{2} W / \partial \beta_{2}^{2} \neq 0$;

2) $(\boldsymbol{t}, \boldsymbol{\beta}) \in \mathcal{M}^{\text {sing }}$, если и только если по крайней мере одна из производных $\partial^{2} W / \partial \beta_{1}^{2}, \partial^{2} W / \partial \beta_{2}^{2}$ обращается в нуль.

Более того, используя уравнения (4), нетрудно получить, что в любой точке $(\boldsymbol{t}, \boldsymbol{\beta}) \in \mathcal{M}$ все смешанные производные $\partial_{\beta_{1}}^{i} \partial_{\beta_{2}}^{j} W$ можно выразить в терминах линейной комбинации производных $\partial_{\beta_{1}}^{n} W$ и $\partial_{\beta_{2}}^{m} W$. Поэтому, определяя $\mathcal{M}_{n_{1}, n_{2}}^{\text {sing }}$ как множество таких точек $(\boldsymbol{t}, \boldsymbol{\beta}) \in \mathcal{M}$, что

$$
\frac{\partial^{n_{i}+2} W}{\partial \beta_{i}^{n_{i}+2}} \neq 0, \quad \frac{\partial^{k} W}{\partial \beta_{i}^{k}}=0 \quad \text { для всех } \quad 1 \leqslant k \leqslant n_{i}+1, \quad i=1,2,
$$

получаем

$$
\mathcal{M}^{\text {sing }}=\bigcup_{n_{1}+n_{2} \geqslant 1} \mathcal{M}_{n_{1}, n_{2}}^{\text {sing }}
$$

где

$$
\mathcal{M}_{n_{1}, n_{2}}^{\text {sing }} \cap \mathcal{M}_{n_{1}^{\prime}, n_{2}^{\prime}}^{\text {sing }}=\varnothing
$$

при $\left(n_{1}, n_{2}\right) \neq\left(n_{1}^{\prime}, n_{2}^{\prime}\right)$. Можно охарактеризовать классы $\mathcal{M}_{n_{1}, n_{2}}^{\text {sing }}$ сингулярного сектора в терминах того, как $S(\lambda)$ ведет себя при $\lambda=\beta_{i}, i=1,2$. Действительно, производная $\partial_{\beta_{1}}^{k+1} W$ при $k \geqslant 1$ пропорциональна интегралу

$$
\oint_{\gamma} \frac{d \lambda}{2 i \pi} \frac{V(\lambda, \boldsymbol{t})}{\left(\lambda-\beta_{1}\right)^{k+1} \sqrt{\left(\lambda-\beta_{1}\right)\left(\lambda-\beta_{2}\right)}}=\oint_{\gamma} \frac{d \lambda}{2 i \pi} \frac{h(\lambda)}{\left(\lambda-\beta_{1}\right)^{k+1}}=\left.\frac{1}{k !}\left(\partial_{\lambda}^{k} h(\lambda)\right)\right|_{\lambda=\beta_{1}},
$$

и аналогичный результат получается для производных $\partial_{\beta_{2}}^{k+1} W$ при $k \geqslant 2$. Как следствие, получаем

ПрЕДЛОЖЕНИЕ 2. Точка $(\boldsymbol{t}, \boldsymbol{\beta}) \in \mathcal{M}$ принадлежит сингулярному классу $\mathcal{M}_{n_{1}, n_{2}}^{\mathrm{sing}}$, если и только если

$$
S(\lambda, \boldsymbol{t}, \boldsymbol{\beta}) \sim\left(\lambda-\beta_{i}\right)^{\left(2 n_{i}+3\right) / 2} \quad \text { npu } \quad \lambda \rightarrow \beta_{i}, \quad i=1,2 .
$$

\section{3. ЯВНОЕ ОПРЕДЕЛЕНИЕ СИНГУЛЯРНЫХ СЕКТОРОВ}

Нетрудно видеть, что сингулярные классы $\mathcal{M}_{n_{1}, n_{2}}^{\text {sing }}$ определяются системой $n_{1}+n_{2}$ связей, наложенных на координаты $\boldsymbol{t}$. Действительно, точки $(\boldsymbol{t}, \boldsymbol{\beta})$ из $\mathcal{M}_{n_{1}, n_{2}}^{\text {sing }}$ характеризуются уравнениями

$$
\frac{\partial^{k} W}{\partial \beta_{i}^{k}}=0 \quad \text { для всех } \quad 1 \leqslant k \leqslant n_{i}+1, \quad i=1,2,
$$

и

$$
\frac{\partial^{n_{i}+2} W}{\partial \beta_{i}^{n_{i}+2}} \neq 0, \quad i=1,2 .
$$

5 Теоретическая и математическая физика, т. 168, №1, 2011 г. 
Теперь заметим, что якобиан матричной системы из двух уравнений

$$
\frac{\partial^{n_{i}+1} W}{\partial \beta_{i}^{n_{i}+1}}=0, \quad i=1,2,
$$

не сингулярен:

$$
\Delta:=\left|\begin{array}{cc}
\frac{\partial^{n_{1}+2} W}{\partial \beta_{1}^{n_{1}+2}} & \frac{\partial^{n_{2}+2} W}{\partial \beta_{1} \partial \beta_{2}^{n_{2}+1}} \\
\frac{\partial^{n_{1}+2} W}{\partial \beta_{1}^{n_{1}+1} \partial \beta_{2}} & \frac{\partial^{n_{2}+2} W}{\partial \beta_{2}^{n_{2}+2}}
\end{array}\right| \neq 0 .
$$

Действительно, отметим, что вследствие уравнений (4) производные вне диагонали в $\Delta$ являются линейными комбинациями производных $\left\{\partial_{\beta_{i}}^{k} W, 1 \leqslant k \leqslant n_{i}+1, i=\right.$ $1,2\}$, из формул $(21),(22)$ получаем

$$
\Delta=\frac{\partial^{n_{1}+2} W}{\partial \beta_{1}^{n_{1}+2}} \frac{\partial^{n_{2}+2} W}{\partial \beta_{2}^{n_{2}+2}} \neq 0 .
$$

Поэтому можно решить систему (23) и получить решение $\boldsymbol{\beta}(\boldsymbol{t})$. Его подстановка в оставшиеся уравнения (21) дает $n_{1}+n_{2}$ связей вида

$$
f_{k}(\boldsymbol{t})=0, \quad k=1, \ldots, n_{1}+n_{2} .
$$

Нетрудно найти решения уравнений (21), (22) в двух простых случаях: с одним параметром $t_{3}\left(t_{4}=t_{5}=\cdots=0\right)$ и с двумя параметрами $t_{3}, t_{4}\left(t_{5}=t_{6}=\cdots=0\right)$. Получаем

$$
\mathcal{M}^{\text {sing }}=\mathcal{M}_{10}^{\text {sing }} \cup \mathcal{M}_{01}^{\text {sing }},
$$

где $\mathcal{M}_{10}^{\text {sing }}$ определяется следующим образом:

$$
\begin{aligned}
1) & x=\frac{-45 t_{4} t_{3}^{3}+180 t_{2} t_{4}^{2} t_{3}+\sqrt{15}\left(8 t_{2} t_{4}-3 t_{3}^{2}\right) \sqrt{t_{4}^{2}\left(3 t_{3}^{2}-8 t_{2} t_{4}\right)}}{360 t_{4}^{3}}, \\
\beta_{1} & =-\frac{5 t_{3} t_{4}+\sqrt{15} \sqrt{t_{4}^{2}\left(3 t_{3}^{2}-8 t_{2} t_{4}\right)}}{20 t_{4}^{2}}, \quad \beta_{2}=\frac{-3 t_{3} t_{4}+\sqrt{15} \sqrt{t_{3}^{2}\left(3 t_{3}^{2}-8 t_{2} t_{4}\right)}}{12 t_{4}^{2}} ; \\
2) \quad x & =\frac{-45 t_{4} t_{3}^{3}+180 t_{2} t_{3}^{2} t_{3}-\sqrt{15}\left(8 t_{2} t_{4}-3 t_{3}^{2}\right) \sqrt{t_{4}^{2}\left(3 t_{3}^{2}-8 t_{2} t_{4}\right)}}{360 t_{4}^{3}} ; \\
\beta_{1} & =\frac{-5 t_{3} t_{4}+\sqrt{15} \sqrt{t_{4}^{2}\left(3 t_{3}^{2}-8 t_{2} t_{4}\right)}}{20 t_{4}^{2}}, \quad \beta_{2}=-\frac{3 t_{3} t_{4}+\sqrt{15} \sqrt{t_{4}^{2}\left(3 t_{3}^{2}-8 t_{2} t_{4}\right)}}{12 t_{4}^{2}} ;
\end{aligned}
$$

$\mathcal{M}_{01}^{\text {sing }}$ определяется как

$$
\begin{aligned}
1) & x=\frac{-45 t_{4} t_{3}^{3}+180 t_{2} t_{4}^{2} t_{3}-\sqrt{15}\left(8 t_{2} t_{4}-3 t_{3}^{2}\right) \sqrt{t_{4}^{2}\left(3 t_{3}^{2}-8 t_{2} t_{4}\right)}}{360 t_{4}^{3}}, \\
\beta_{1} & =-\frac{3 t_{3} t_{4}+\sqrt{15} \sqrt{t_{4}^{2}\left(3 t_{3}^{2}-8 t_{2} t_{4}\right)}}{12 t_{4}^{2}}, \quad \beta_{2}=\frac{-5 t_{3} t_{4}+\sqrt{15} \sqrt{t_{4}^{2}\left(3 t_{3}^{2}-8 t_{2} t_{4}\right)}}{20 t_{4}^{2}} ; \\
2) \quad x & =\frac{-45 t_{4} t_{3}^{3}+180 t_{2} t_{4}^{2} t_{3}+\sqrt{15}\left(8 t_{2} t_{4}-3 t_{3}^{2}\right) \sqrt{t_{4}^{2}\left(3 t_{3}^{2}-8 t_{2} t_{4}\right)}}{360 t_{4}^{3}}, \\
\beta_{1} & =\frac{-3 t_{3} t_{4}+\sqrt{15} \sqrt{t_{4}^{2}\left(3 t_{3}^{2}-8 t_{2} t_{4}\right)}}{12 t_{4}^{2}}, \quad \beta_{2}=-\frac{5 t_{3} t_{4}+\sqrt{15} \sqrt{t_{4}^{2}\left(3 t_{3}^{2}-8 t_{2} t_{4}\right)}}{20 t_{4}^{2}} .
\end{aligned}
$$




\section{4. СИНГУЛЯРНЫЙ СЕКТОР ЭЛЛИПТИЧЕСКОЙ В(1)-СИСТЕМЫ}

Теперь рассмотрим эллиптическую $B(1)$-систему (1). Сингулярный сектор $\mathcal{M}^{\text {sing }}$ в этом случае имеет структуру, отличную от таковой для гиперболической системы. Действительно, поскольку $\beta_{1}=\bar{\beta}_{2}$, функция $W$ при вещественных $x, t_{2}, t_{3}, \ldots$ является вещественнозначной:

$$
\overline{W(\boldsymbol{t}, \beta, \bar{\beta})}=W(\boldsymbol{t}, \beta, \bar{\beta}),
$$

и уравнение годографа (3) имеет вид условия Коши-Римана $\left(\beta=\beta_{1}\right)$ :

$$
\frac{\partial W}{\partial \bar{\beta}}=0
$$

Регулярный и сингулярный секторы $\mathcal{M}^{\text {reg }}$ и $\mathcal{M}^{\operatorname{sing}}$ определяются как такие множества $(\boldsymbol{t}, \beta, \bar{\beta})$ решений уравнения (25), что эрмитова форма

$$
d^{2} W=\frac{\partial^{2} W}{\partial \beta^{2}} d \beta^{2}+2 \frac{\partial^{2} W}{\partial \beta \partial \bar{\beta}} d \beta d \bar{\beta}+\frac{\partial^{2} W}{\partial \bar{\beta}^{2}} d \bar{\beta}^{2}
$$

является соответственно невырожденной или вырожденной. Для нередуцированных решений $(\beta \neq \bar{\beta})$ из соответствующего уравнения ЭПД следует, что

$$
\frac{\partial^{2} W}{\partial \beta \partial \bar{\beta}}=0
$$

поэтому

$$
\left|\begin{array}{ll}
\frac{\partial^{2} W}{\partial \beta^{2}} & \frac{\partial^{2} W}{\partial \beta \partial \bar{\beta}} \\
\frac{\partial^{2} W}{\partial \beta \partial \bar{\beta}} & \frac{\partial^{2} W}{\partial \bar{\beta}^{2}}
\end{array}\right|=\left|\frac{\partial^{2} W}{\partial \bar{\beta}^{2}}\right|^{2}
$$

Таким образом, имеем

ПреДЛОЖЕНИЕ 3. Для нередуиированых решений эллиптической B(1)-системы регулярный сектор $\mathcal{M}^{\text {reg }}$ определяется условием

$$
\frac{\partial W}{\partial \bar{\beta}}=0, \quad \frac{\partial^{2} W}{\partial \bar{\beta}^{2}} \neq 0
$$

Анализ, аналогичный тому, что был проделан для гиперболического случая, быстро приводит к следующему предложению

ПРЕДЛОЖЕНИЕ 4. Сингулярный сектор $\mathcal{M}^{\text {sing }}$ эллиптической $B(1)$-системы (1) представляет собой объединение подпространств $\mathcal{M}_{n}^{\text {sing }}, n=1,2,3, \ldots$, определенHox как

$$
\mathcal{M}_{n}^{\text {sing }}=\left\{(\boldsymbol{t}, \beta, \bar{\beta}) \in \mathcal{M}^{\text {sing }}: \frac{\partial^{k} W}{\partial \bar{\beta}^{k}}=0, k=1, \ldots, n+1 ; \frac{\partial^{(n+2)} W}{\partial \bar{\beta}^{(n+2)}} \neq 0\right\} .
$$

Решения, принадлежащие $\mathcal{M}_{n}^{\text {sing }}$, определенъ на подпространстве коразмерности $2 n$ в пространстве параметров $x, t_{2}, t_{3}, \ldots$. 
Таким образом, в эллиптическом случае градиентная катастрофа наступает в точке $(x, t)$ при фиксированных параметрах $t_{2}, t_{3}, \ldots$. Аналогично гиперболическому случаю подпространство $\mathcal{M}_{n}^{\text {sing }}$ не пусто, если по крайней мере $n$ параметров $t_{2}, t_{3}, \ldots, t_{n+1}$ отличны от нуля в формуле $(13)$.

Полезно переписать формулу (13) для функции $W$ в терминах вещественной и мнимой частей $\beta_{1}$, т. е. $\beta_{1}=U+i V$ :

$$
\begin{aligned}
W=x U & +t_{2}\left(U^{2}-\frac{1}{2} V^{2}\right)+t_{3}\left(U^{3}-\frac{3}{2} U V^{2}\right)+t_{4}\left(U^{4}-3 U^{2} V^{2}+\frac{3}{8} V^{4}\right)+ \\
& +t_{5}\left(U^{5}-5 U^{3} V^{2}+\frac{15}{8} U V^{4}\right)+\cdots
\end{aligned}
$$

Эта формула явно показывает характер эллиптических сингулярностей, проявляющихся для функции $W$ при различных значениях параметров $t_{2}, t_{3}, \ldots$.

Основные уравнения (13), (25), а также условия (28), определяющие подпространство $\mathcal{M}_{n}^{\text {sing }}$, легко переписать в терминах исходных переменных $u$ и $v$. Поскольку

$$
\frac{\partial W}{\partial \beta}=-\frac{\partial W}{\partial u}+i \sqrt{-v} \frac{\partial W}{\partial v}
$$

уравнение годографа (25) при $v \neq 0$ принимает вид

$$
\frac{\partial W}{\partial u}=0, \quad \frac{\partial W}{\partial v}=0,
$$

в то время как уравнение ЭПД и уравнение (30) имеют вид

$$
\frac{\partial^{2} W}{\partial u^{2}}-v \frac{\partial^{2} W}{\partial v^{2}}=0
$$

Для подпространства $\mathcal{M}_{1}^{\text {sing }}$ условия (28) записываются как

$$
\frac{\partial W}{\partial \beta}=0, \quad \frac{\partial^{2} W}{\partial \beta^{2}}=0, \quad \frac{\partial^{3} W}{\partial \beta^{3}} \neq 0 .
$$

Поскольку

$$
\frac{\partial^{2} W}{\partial \beta^{2}}=\frac{\partial^{2} W}{\partial u^{2}}-2 i \sqrt{-v} \frac{\partial^{2} W}{\partial u \partial v}+v \frac{\partial^{2} W}{\partial v^{2}}+\frac{1}{2} \frac{\partial W}{\partial v},
$$

с учетом уравнений (30) и (31) можно заключить, что второе условие (32) выполнено, если и только если

$$
\frac{\partial^{2} W}{\partial u^{2}}=0, \quad \frac{\partial^{2} W}{\partial v^{2}}=0, \quad \frac{\partial^{2} W}{\partial u \partial v}=0 .
$$

Таким образом, подпространство $\mathcal{M}_{1}^{\text {sing }}$ характеризуется условиями (30), (33) и требованием, чтобы производные $W$ третьего порядка были отличны от нуля.

Чтобы сравнить эти условия с условиями из работы [4], заметим сначала, что $B(1)$-система (1) переходит в систему (1.8) при подстановке $u \rightarrow v, v \rightarrow-u$. Тогда, если выбрать

$$
W=f(u, v)+x v-u v t
$$


то уравнения годографа (30) принимают вид уравнений (2.4) из работы [4], а уравнение (31) редуцируется к уравнению (2.5) из той же работы. Наконец, при таком выборе условия (33) переходят в условие (2.12) из работы [4].

Наконец, заметим, что согласно предложению 4 для подпространства $\mathcal{M}_{1}^{\text {sing }}$ коразмерность соответствующего подпространства $\left(x, t_{2}, t_{3}, \ldots\right)$ равна двум, а функция $W$ при $t_{n}=0, n \geqslant 4$, т. е.

$$
W=x U+t_{2}\left(U^{2}-\frac{1}{8} V^{2}\right)+t_{3}\left(U^{3}-\frac{3}{2} U V^{2}\right),
$$

проявляет эллиптическую омбилическую сингулярность согласно классификации Тома [17] (см., например, работы [16], [18], [19]). Эти результаты воспроизводят формулы, впервые полученные в работе [4] (формула (4.2)).

\section{5. БЕЗДИСПЕРСИОННАЯ ИЕРАРХИЯ ТОДЫ}

Теперь рассмотрим функцию

$$
W_{\mathrm{T}}\left(x, \beta_{1}, \beta_{2}\right)=\int \frac{d \lambda}{2 \pi i} V_{\mathrm{T}}(x, \lambda) \sqrt{\left(1-\frac{\beta_{1}}{\lambda}\right)\left(1-\frac{\beta_{2}}{\lambda}\right)},
$$

где $V_{\mathrm{T}}(x, \lambda)=\sum_{n \geqslant 0} \lambda^{n} x_{n}$. Критические точки для этой функции определяются уравнениями

$$
\frac{\partial W_{\mathrm{T}}}{\partial \beta_{1}}=0, \quad \frac{\partial W_{\mathrm{T}}}{\partial \beta_{2}}=0 .
$$

Простая проверка показывает, что $W_{\mathrm{T}}$ удовлетворяет уравнению ЭПД типа $E(-1 / 2,-1 / 2)$ :

$$
2\left(\beta_{1}-\beta_{2}\right) \frac{\partial^{2} W_{\mathrm{T}}}{\partial \beta_{1} \partial \beta_{2}}=-\left(\frac{\partial W_{\mathrm{T}}}{\partial \beta_{1}}-\frac{\partial W_{\mathrm{T}}}{\partial \beta_{2}}\right) .
$$

В явной записи функция $W_{\mathrm{T}}$ представляет собой ряд:

$$
\begin{aligned}
W_{\mathrm{T}}= & -\frac{1}{2} x_{0}\left(\beta_{1}+\beta_{2}\right)-\frac{1}{8} x_{1}\left(\beta_{1}-\beta_{2}\right)^{2}-\frac{1}{16} x_{2}\left(\beta_{1}+\beta_{2}\right)\left(\beta_{1}-\beta_{2}\right)^{2}- \\
& -\frac{1}{128} x_{3}\left(5 \beta_{1}^{2}+6 \beta_{1} \beta_{2}+5 \beta_{1}^{2}\right)\left(\beta_{1}-\beta_{2}\right)^{2}+\cdots,
\end{aligned}
$$

а уравнения годографа принимают вид

$$
\begin{aligned}
& x_{0}+\frac{1}{2} x_{1}\left(\beta_{1}-\beta_{2}\right)+\frac{1}{8} x_{2}\left(3 \beta_{1}^{2}-2 \beta_{1} \beta_{2}-\beta_{2}^{2}\right)+\cdots=0, \\
& x_{0}-\frac{1}{2} x_{1}\left(\beta_{1}-\beta_{2}\right)+\frac{1}{8} x_{2}\left(3 \beta_{2}^{2}-2 \beta_{1} \beta_{2}-\beta_{1}^{2}\right)+\cdots=0 .
\end{aligned}
$$

Эти уравнения дают решения системы

$$
\frac{\partial \beta_{1}}{\partial x_{1}}=\frac{1}{2}\left(\beta_{1}-\beta_{2}\right) \frac{\partial \beta_{1}}{\partial x_{0}}, \quad \frac{\partial \beta_{2}}{\partial x_{1}}=-\frac{1}{2}\left(\beta_{1}-\beta_{2}\right) \frac{\partial \beta_{2}}{\partial x_{0}} .
$$

В терминах переменных $u=-\left(\beta_{1}+\beta_{2}\right), v=\left(\beta_{1}-\beta_{2}\right)^{2} / 4$ получаем бездисперсионную систему Тоды (2). Рассматривая высшие времена $x_{2}, x_{3}, \ldots$, получаем полную бездисперсионную иерархию Тоды. 
Аналогично случаю Бенни функция $W_{\mathrm{T}}$ является производящей функцией для классических сингулярностей функций двух переменных. Действительно, в переменных $X=\left(\beta_{1}+\beta_{2}\right) / 2, Y=\left(\beta_{1}-\beta_{2}\right) / 2$ она имеет вид

$$
W_{\mathrm{T}}=-x_{0} X-\frac{1}{2} x_{1} Y^{2}-\frac{1}{2} x_{2} X Y^{2}-\frac{1}{8} x_{3}\left(4 X^{2}+Y^{2}\right) Y^{2}+\cdots,
$$

где третий член представляет параболическую омбилическую особенность как для гиперболического, так и для эллиптического случаев.

Представленные здесь формулы для бездисперсионной иерархии Тоды совпадают с формулами, полученными в работе [20], после отождествления

$$
V_{\mathrm{T}}(x, \lambda)=-2 T \lambda+\lambda V_{\mathrm{H}}^{\prime}(t, \lambda),
$$

т. е. $x_{0}=-2 T, x_{n}=n t_{n}, n=1,2,3, \ldots$.

Очевидно, что описание регулярного и сингулярного секторов для бездисперсионной иерархии Тоды полностью совпадает с описанием соответствующих секторов для однослойной иерархии Бенни.

\section{6. ВЗАИМНЫЕ СООТНОШЕНИЯ МЕЖДУ УРАВНЕНИЯМИ ЭПД С РАЗЛИЧНЫМИ ИНДЕКСАМИ, А ТАКЖЕ ДЛЯ ФУНКЦИИ $W$ И ПЛОТНОСТЕЙ ИНТЕГРАЛОВ ДВИЖЕНИЯ}

Однослойная иерархия Бенни и бездисперсионная иерархия Тоды - два примера систем гидродинамического типа, для которых функции $W$ удовлетворяют уравнениям ЭПД

$$
L_{\varepsilon} W_{\varepsilon}:=\left[\frac{\partial^{2}}{\partial x \partial y}-\frac{\varepsilon}{x-y}\left(\frac{\partial}{\partial x}-\frac{\partial}{\partial y}\right)\right] W_{\varepsilon}=0
$$

с различными индексами $\varepsilon$. Такие линейные уравнения хорошо изучены (см., например, монографию [8]). У операторов $L_{\varepsilon}$ есть несколько замечательных свойств. Одно из них (возможно, ранее пропущенное) определяется тождеством

$$
L_{\varepsilon+1} L_{\mu}=L_{\mu+1} L_{\varepsilon}
$$

для произвольных индексов $\varepsilon$ и $\mu$. Из этого тождества, наример, следует, что для любого решения $W_{\varepsilon}$ функция $L_{\mu} W_{\varepsilon}$ с произвольным $\mu$ удовлетворяет уравнению ЭПД с индексом $\varepsilon+1$, более точно, $L_{\mu} W_{\varepsilon}=\varepsilon(\varepsilon-\mu) W_{\varepsilon+1}$. В частности, при $\varepsilon=-1 / 2$ и $\mu=0$ получаем $L_{1 / 2} L_{0}=L_{1} L_{-1 / 2}$. В терминах операторов $\tilde{L}_{\varepsilon}$, определяемых как $\tilde{L}_{\varepsilon}=(x-y) L_{\varepsilon}$, последнее соотношение принимает вид

$$
\partial_{x} \partial_{y} \tilde{L}_{-1 / 2}=\tilde{L}_{1 / 2} \partial_{x} \partial_{y}
$$

Это тождество ясно демонстрирует дуальность между уравнениями ЭПД с индексами $1 / 2$ и $-1 / 2$ и, следовательно, между однослойной иерархией Бенни и бездисперсионной иерархией Тоды.

Дуальность между функциями $W$ и плотностями интегралов движения является дуальностью другого типа, типичного для так называемых $\varepsilon$-интегрируемых систем 
гидродинамического типа. Действительно, в соответствии с результатом Царева [21] симметрия $w_{i}$ полугамильтоновой гидродинамической системы

$$
\frac{\partial \beta_{i}}{\partial t}=\lambda_{i}(\beta) \frac{\partial \beta_{i}}{\partial x}, \quad i=1, \ldots, n,
$$

т. е. решение системы

$$
\frac{\partial \beta_{i}}{\partial \tau}=w_{i}(\beta) \frac{\partial \beta_{i}}{\partial x}, \quad i=1, \ldots, n,
$$

которая коммутирует с системой (44), определяется системой

$$
\frac{\partial w_{k} / \partial \beta_{i}}{w_{i}-w_{k}}=\frac{\partial \lambda_{k} / \partial \beta_{i}}{\lambda_{i}-\lambda_{k}}, \quad i \neq k .
$$

Такие $w_{i}$ обеспечивают нас решениями систем (44) посредством уравнений годографа:

$$
\Omega_{i}:=-x+\lambda_{i}(\beta) t+w_{i}=0, \quad i=1, \ldots, n .
$$

Для такой системы (44) плотности $P$ интегралов движения удовлетворяют уравнениям [21]

$$
\frac{\partial^{2} P}{\partial \beta_{i} \partial \beta_{k}}=\frac{\partial \lambda_{i} / \partial \beta_{k}}{\lambda_{i}-\lambda_{k}} \frac{\partial P}{\partial \beta_{i}}-\frac{\partial \lambda_{k} / \partial \beta_{i}}{\lambda_{i}-\lambda_{k}} \frac{\partial P}{\partial \beta_{k}}, \quad i \neq k .
$$

Определим $\varepsilon$-системы как системы (особый класс таких систем см., например, в работе [22]), для которых

$$
\frac{\partial \lambda_{i} / \partial \beta_{k}}{\lambda_{i}-\lambda_{k}}=\frac{\partial \lambda_{k} / \partial \beta_{i}}{\lambda_{i}-\lambda_{k}}=\frac{\varepsilon}{\beta_{i}-\beta_{k}} .
$$

Для таких систем плотности интегралов удовлетворяют уравнениям ЭПД:

$$
\frac{\partial^{2} P}{\partial \beta_{i} \partial \beta_{k}}=\frac{\varepsilon}{\beta_{i}-\beta_{k}} \frac{\partial P}{\partial \beta_{i}}-\frac{\varepsilon}{\beta_{i}-\beta_{k}} \frac{\partial P}{\partial \beta_{k}}, \quad i \neq k .
$$

В то же время уравнения для $w_{i}$ принимают вид

$$
\frac{\partial w_{k}}{\partial \beta_{i}}=-\varepsilon \frac{w_{i}-w_{k}}{\beta_{i}-\beta_{k}}, \quad i \neq k .
$$

Из симметрии этих уравнений относительно перестановки индексов $i$ и $k$ следует, что $\partial w_{k} / \partial \beta_{i}=\partial w_{i} / \partial \beta k$, поэтому

$$
w_{i}=\frac{\partial \widetilde{W}}{\partial \beta_{i}}, \quad i=1, \ldots, n,
$$

для некоторой функции $\widetilde{W}$. Таким образом, уравнения (51) представляют собой уравнения ЭПД типа $E(-\varepsilon,-\varepsilon)$ для функции $\widetilde{W}$ :

$$
\frac{\partial^{2} \widetilde{W}}{\partial \beta_{i} \partial \beta_{k}}=-\left(\frac{\varepsilon}{\beta_{i}-\beta_{k}} \frac{\partial \widetilde{W}}{\partial \beta_{i}}-\frac{\varepsilon}{\beta_{i}-\beta_{k}} \frac{\partial \widetilde{W}}{\partial \beta_{k}}\right), \quad i \neq k .
$$


Тот факт, что производящие функции для симметрий в уравнениях Уизема и некоторых других интегрируемых гидродинамических систем удовлетворяют уравнениям ЭПД, был отмечен ранее в работах [9], [22], [23]. Дуальность между уравнениями ЭПД для плотностей интегралов движения и производящих функций симметрий также уже отмечалась. Однако приведенное выше доказательство представляется отличным от обсуждавшихся ранее.

Кроме того, можно отметить следующее: из уравнений (49) следует, что для $\varepsilon$-систем также $\lambda_{i}=\partial g / \partial \beta_{i}$ для некоторой функции $g$. В результате этого уравнения годографа (47) для $\varepsilon$-систем принимают вид

$$
\Omega_{i}=-x+t \frac{\partial g}{\partial \beta_{i}}+\frac{\partial \widetilde{W}}{\partial \beta_{i}}=\frac{\partial W}{\partial \beta_{i}}=0, i, \ldots, n,
$$

где $W=-x\left(\beta_{1}+\beta_{2}\right)+g t+\widetilde{W}$. Таким образом, уравнения годографа для интегрируемых систем гидродинамического типа являются не чем иным, как уравнениями, определяющими критические точки функции $W$. По-видимому, этот факт был упущен в предыдущих публикациях. Более того, согласно уравнениям (49) функция $g$ также удовлетворяет уравнению ЭПД $E(-\varepsilon,-\varepsilon)$, и поэтому то же верно для функции $W$. Заметим, что $\varepsilon$-системы особого класса, для которых $\lambda_{i}$ являются линейными функциями $\beta_{i}$, рассматривались ранее в работе [22].

Таким образом, для интегрируемых $\varepsilon$-систем гидродинамического типа плотности интегралов и функций $W$ (а также функций $\widetilde{W}$ производящих симметрии) играют двойственную роль, удовлетворяя уравнениям ЭПД с противоположным знаком индекса $\varepsilon$. Это свойство очень похоже на хорошо известную дуальность между производящими функциями интегралов движения и симметриями для интегрируемых уравнений с дисперсией.

Благодарности. Авторы благодарят Ministerio de Educación y Ciencia Италии (исследовательский проект FIS2008-00200/FIS).

\section{Список литературы}

[1] Б. А. Дубровин, С. П. Новиков, УМН, 44:6(270) (1989), 29-98.

[2] D. J. Benney, Stud. Appl. Math., 52 (1973), 45-50.

[3] В. Е. Захаров, Функи. анализ и его прил., 14:2 (1980), 15-24.

[4] B. Dubrovin, T. Grava, C. Klein, Nonlinear Sci., 19:1 (2009), 57-94, arXiv: 0704.0501.

[5] C. P. Boyer, J. D. Finley, J. Math. Phys., 23:6 (1982), 1126-1130.

[6] M. Mineev-Weinstein, P. B. Wiegmann, A. Zabrodin, Phys. Rev. Lett., 84:22 (2000), 5106-5109, arXiv: nlin/0001007.

[7] L. Martínez Alonso, E. Medina, J. Phys. A, 41:33 (2008), 335202, 17 pp., arXiv: 0804.3498.

[8] G. Darboux, Leçons sur la théorie générale des surfaces, v. II, Gauthier Villars, Paris, 1915.

[9] V.R. Kudashev, S. E. Sharapov, Phys. Lett. A, 154:9 (1991), 445-448; В. Р. Кудашев, C. Е. Шарапов, $T M \Phi, ~ 87: 1$ (1991), 40-47.

[10] F. R. Tian, Comm. Pure Appl. Math., 46 (1993), 1093-1129.

[11] F. R. Tian, Duke Math. J., 74:1 (1994), 203-221.

[12] Y. Kodama, B. G. Konopelchenko, J. Phys. A, 35:31 (2002), L489-L500, arXiv: nlin/0205012.

[13] B. G. Konopelchenko, L. Martínez Alonso, J. Phys. A, 37:32 (2004), 7859-7877. 
[14] I. M. Krichever, Comm. Pure Appl. Math., 47:4 (1994), 437-475.

[15] B. G. Konopelchenko, L. Martínez Alonso, E. Medina, J. Phys. A, 43:43 (2010), 434020, 15 pp., arXiv: 1003.2892.

[16] Л. Альфорс, Лекиии по квазиконформным отображениям, Мир, М., 1969.

[17] R. Thom, Structural Stability and Morphogenesis. An Outline of a General Theory of Models, Adv. Book Class., Addison-Wesley, Redwood City, CA, 1989.

[18] В. И. Арнольд, Функи. анализ и его прил., 6:4 (1972), 3-25; УМН, 30:5(185) (1975), 3-65.

[19] В. И. Арнольд, А.Н. Варченко, С. М. Гусейн-Заде, Особенности дифферениируемых отображений, т. 1, 2, Наука, М., 1982.

[20] B. Konopelchenko, L. Martínez Alonso, E. Medina, Phys. Lett. A, 375:5 (2011), 867-872, arXiv: 1005.4773.

[21] С. П. Царев, Докл. АН СССР, 282:3 (1985), 534-537.

[22] M. V. Pavlov, J. Math. Phys., 44:9 (2003), 4134-4156, arXiv: nlin/0301010.

[23] М.В. Павлов, Гамильтонов формализм уравнений электрофореза. Интегрируемые уравнения гидродинамики, Препринт № 17, ИТФ, М., 1987. 